\title{
Integrated inverse design of ventilation for an aircraft cabin
}

\author{
Yun $\mathrm{Wei}^{1}$, Wei $\mathrm{Liu}^{2}$, Yu Xue ${ }^{1}$, Zhiqiang (John) Zhai ${ }^{1,3}$, Qingyan (Yan) Chen ${ }^{4}$, and Tengfei (Tim) Zhang ${ }^{1,5,6, *}$ \\ ${ }^{1}$ School of Civil Engineering, Dalian University of Technology, Dalian 116024, China \\ ${ }^{2}$ Department of Civil and Architectural Engineering, KTH Royal Institute of Technology, Stockholm 100 44, Sweden \\ ${ }^{3}$ Civil, Environmental and Architectural Engineering, University of Colorado, Boulder, CO 80309, USA \\ ${ }^{4}$ School of Mechanical Engineering, Purdue University, West Lafayette, IN 47907, USA \\ ${ }^{5}$ Tianjin Key Laboratory of Indoor Air Environmental Quality Control, School of Environmental Science and Engineering, \\ Tianjin University, 92 Weijin Road, Tianjin 300072, China \\ ${ }^{6}$ Key Laboratory of Ocean Energy Utilization and Energy Conservation of Ministry of Education, Dalian University of \\ Technology (DUT), 2 Linggong Road, Dalian 116024, China
}

\begin{abstract}
Cabin ventilation is crucial for maintaining thermal comfort and air quality for passengers and crew. The genetic algorithm, proper orthogonal decomposition (POD), and adjoint method have been proposed to inversely design the cabin ventilation. However, each method has its cons and pros. This paper proposed to integrate the above three methods in cascades. The genetic algorithm was applied first in the first stage to roughly circumscribe the ranges of design parameters. Then POD was applied in the next stage to further narrow the ranges and estimate the optimal parametric sets for each design criterion. The estimated optimal design from POD was supplied to the adjoint method for fine tuning. The air-supply parameters of a five-row aircraft cabin were inversely designed to achieve the minimum absolute value of the predicted mean vote (PMV) and the minimum averaged mean age of air. The results showed that the integrated method was able to improve the design stage by stage. The integrated method has superior advantages to assure the optimal design while minimizing the computing expense.
\end{abstract}

\section{Introduction}

Cabin ventilation is crucial for maintaining thermal comfort and air quality for passengers and crew. Design of cabin ventilation must get the air-supply parameters appropriately determined. The conventional design adopts an iterative guess-and-correction procedure. The initial air-supply parameters are guessed, and the corresponding comfort and air quality performances are solved. If the design targets are not obtained, the airsupply parameters must be gradually adjusted, until the design objectives are satisfied. For contrast, an inverse design starts from the design targets of cabin environmental performances, and the required air-supply parameters are provided in one-shot process. In recent years, inverse methods, such as the CFD-based genetic algorithm, CFD-based proper orthogonal decomposition (POD), and CFD-based adjoint method, have been proposed.

The CFD-based genetic algorithm imitates the natural evolution to search for the optimal design from the randomly initial generations. With "crossing" and "mutation", only superior cases with their environmental performances closer to the targets are selected to form the next generation. The genetic algorithm was proposed in the 1970s for major applications in biology, control, and artificial intelligence [1], and since then many researchers [2-3] sought to improve the algorithm. Xue et al. [4] and Zhai et al. [5] applied the method to inversely determine the air-supply parameters for cabin ventilation. The genetic algorithm is robust to provide the globally optimal solution. However, the method is resource-demanding and thus the design efficiency is low.

The POD establishes a quick map of cabin environmental performance from various design parameters. By specifying the expected thermal comfort and air quality, the design parameters satisfying the targets are promptly determined. The POD first extracts the POD modes containing dominant thermo-flow features from some characteristic design cases. Then the thermo-flow distributions for remaining cases are solved by interpolating the coefficients of the POD modes. The POD was originally proposed to predict turbulent flows [6]. Recently, the POD was adopted to inversely design the air-supply opening size, air-discharge direction, and air temperature [7-8]. The POD-based method is efficient. However, the solution may not be very accurate due to the model reduction.

The adjoint method searches for an optimal design from a specified initial case by means of a gradientbased optimization. Different programs have been developed to implement the adjoint method in optimizing heat transfer [9], geometric shapes [10], and identifying pollutant sources [11]. The adjoint method has been utilized to inversely design air-supply parameters for cabin ventilation [12]. An optimal design can commonly be completed in fewer than 10 design

\footnotetext{
* Corresponding author: timzhang@tju.edu.cn
} 
cycles if the initial design variables are provided within a reasonable range. However, only a local optimal solution can be provided by the method.

Table 1. Pros and cons of the three inverse design methods.

\begin{tabular}{|c|l|l|}
\hline Method & \multicolumn{1}{|c|}{ Pros } & \multicolumn{1}{c|}{ Cons } \\
\hline $\begin{array}{c}\text { Genetic } \\
\text { algorithm }\end{array}$ & $\begin{array}{l}\text { Globally optimal } \\
\text { solution }\end{array}$ & $\begin{array}{l}\text { Low computing } \\
\text { efficiency }\end{array}$ \\
\hline $\begin{array}{c}\text { POD } \\
\text { method }\end{array}$ & $\begin{array}{l}\text { High computing } \\
\text { efficiency }\end{array}$ & Not very accurate \\
\hline $\begin{array}{c}\text { Adjoint } \\
\text { method }\end{array}$ & Accurate & Local optimal solution \\
\hline
\end{tabular}

None of the existent methods are satisfactory enough. Pros and cons of the three methods are summarized in Table 1. If some of the methods are integrated together, they may complement each other. For instance, Chen et al. [8] integrated the genetic algorithm and adjoint method, in which the genetic algorithm provided a rough design while the adjoint method tuned the design. Li et al. [13] integrated the POD and genetic algorithm. The POD was applied first to construct a number of discrete cases and then the genetic algorithm was used to search for an optimal design. The integration seems favourable, however, no systematic justification for the integration has been carried out.

This study proposed an integration of the three methods for inverse design of the air-supply parameters in an aircraft cabin. The genetic algorithm was adopted first to circumscribe ranges of the air-supply parameters. Then POD was used to further narrow the ranges and estimate the optimal design. The adjoint method was finally utilized to tune the design. The conducted designs in each stage were compared, and the justification for method integration was analyzed.

\section{Methodology}

The integrated design includes three stages: an initial design with the CFD-based genetic algorithm, a basic design with the CFD-based POD method, and a fine design with the CFD-based adjoint method. The principles of each stage are outlined below, followed by a procedure for method integration.

\subsection{Initial design}

The initial design with the genetic algorithm starts with numbers of cases with different initial air-supply parameters. These cases are called the first generation of design. The environmental performances of the cases are computed to select superior cases with the performance closer to the target. With "crossing" or "mutating" among each other, the next generation is produced. The process is cycled until either a maximum number of generations or the set design targets are reached. Then the optimization is terminated, and the circumscribed range of parametric design is outputted.

Note that the objective of the initial design is to circumscribe the parametric range(s) containing the globally optimal design, rather than to produce the globally optimal design directly. The genetic algorithm can simply eliminate those designs that are too far away from the target and just provide the circumscribed parametric ranges for subsequent stages. Consequently, the computing burden of the genetic algorithm is greatly reduced.

\subsection{Basic design}

In the basic design, POD is adopted to further narrow the parametric ranges provided by the genetic algorithm. The POD modes are first extracted from the characteristic cases solved by full CFD simulations as:

$$
\boldsymbol{\varphi}=\sum_{j=1}^{N_{v}} \mathbf{f}^{j} a_{j}
$$

where $\boldsymbol{\varphi}$ is the POD mode, $N_{v}$ is the number of representative cases, the superscript $j$ represents the $j^{\text {th }} \mathbf{f}$ in the ensemble of the thermo-flow data, $a_{j}$ is a coefficient that can be solved from the eigenvector of the averaged autocorrelation matrix of the data ensemble. The subscript $j$ is the index of the element in the vector $\mathbf{a}$. The coefficients of the corresponding POD modes can then be solved as:

$$
c_{k}=\left(\mathbf{f}, \varphi^{k}\right)
$$

where (,) is the inner product operation, superscript $k$ represents the $k^{\text {th }}$ POD mode, and subscript $k$ represents the $k^{\text {th }}$ element of the vector c. To obtain the thermo-flow data for the rest air-supply parameters that are not used to extract the POD modes, an interpolation to the coefficients, such as by the piecewise cubic Hermite scheme, can be used. Then the thermo-flow fields in the parametric range can be constructed as:

$$
\mathbf{f}_{\mathbf{R}}=\sum_{k=1}^{K} c_{k} \boldsymbol{\varphi}^{k}
$$

where $\mathbf{f}_{\mathrm{R}}$ is the reconstructed thermo-flow fields, $K$ is the number of POD modes required. Once all of the thermoflow fields in the circumscribled parametric ranges are obtained, the cabin environmental performances can be easily solved. The designs satisfying each design target can be determined, and the ranges of air-supply parameters satisfying all of the design targets can also be obtained by intersecting the designs for each target.

If the characteristic cases are sufficiently representative, the calculated environmental performances from the POD construction can be close to those from full CFD-simulated cases. However, because of model reduction, the solutions provided by POD may not be sufficiently accurate, and thus require some other methods for fine tuning.

\subsection{Fine design}

The optimal design corresponding to each design target obtained by POD is supplied to the adjoint method as the initial case for fine tuning. The adjoint method first 
solves the Navier-Stokes equations to obtain the environmental performance for the current design. Then the adjoint equations of the Navier-Stokes equations are solved to search the direction for gradually minimizing the objective function $O$. The adjoint equations are derived from the gradient to minimize the objective function $O$ for an air-supply parameter $\xi$. The objective function is converted into an unconstrained format as:

$$
L(\xi)=O(\xi)+\int_{\omega}\left(p_{a}, \mathbf{U}_{\mathrm{a}}, T_{a}, \phi_{a}\right) \cdot \mathbf{N} d \omega
$$

where $p_{a}$ is adjoint pressure, $\mathbf{U}_{\mathbf{a}}$ is adjoint velocity, $T_{a}$ is adjoint temperature, $\phi_{a}$ is adjoint scalar, $\omega$ is the solution domain, and $\mathbf{N}$ is a vector that represents the N-S equations. When $\mathbf{N}=\mathbf{0}, L$ is equal to $O$ in Eq. (4). Therefore, finding $\xi$ that minimizes $O$ becomes finding $\xi$ that minimizes $L$. Due to the nonlinearity of objective function, the gradient to minimize $L$ has to be solved for several times to find the optimal solution. In a design cycle both the Navier-Stokes and adjoint equations are solved once.

Because the adjoint method employs full CFD simulation, there is no error from model reduction as the POD method. If the optimal design from the POD is not far from the globally optimal solution, the final accurate solution will be quickly obtained by the adjoint method. More details of the above three methods can be found in [8].

\subsection{Procedure for integrating three methods}

Fig. 1 illustrates the procedure for integrating the three methods. To start design process, the design targets were set and the parametric ranges of the design variables were defined. In the initial design, a generation within the given initial parametric ranges was created. Next, CFD was run to compute the value of the objective function for each case, and the genetic operations were applied to create the next generation until acceptable parametric ranges that were narrower than the initial ones were circumscribed. In the second stage, PODbased basic design was conducted. Representative cases were selected within the circumscribed ranges provided by the genetic algorithm. POD was then applied on the thermo-flow data from full CFD simulations to extract the POD modes. The ranges of design variables that satisfied all of the design targets were identified. The globally optimal design in perspective of each design target was also identified. Finally, the adjoint-based fine design was carried out to tune the optimal parametric sets.

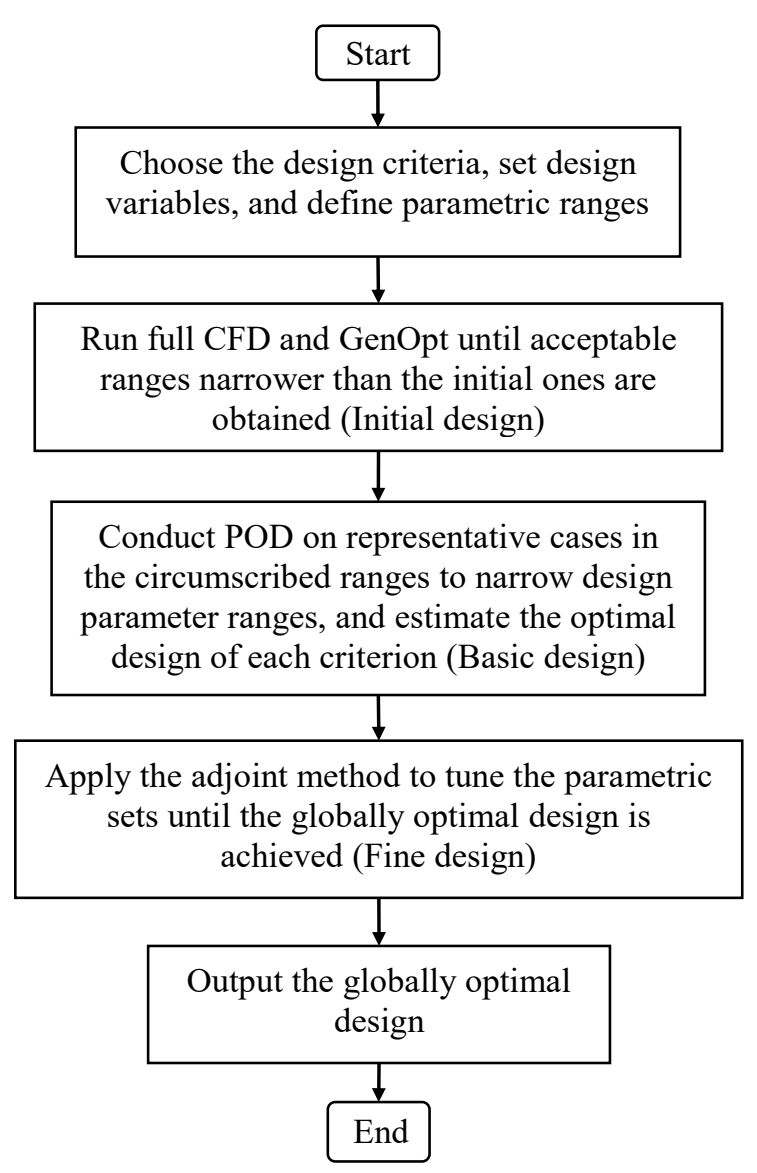

Fig. 1. Solution procedure for integrating three methods.

\section{Demonstration case}

To evaluate the proposed method, the air-supply parameters for a five-row aircraft cabin, as shown in Fig. 2 , were inversely designed. To reduce the computational load, only half of the five-row cabin was modelled with symmetric boundary conditions for the middle sectional plane. Such simplifications would have little effect on the flow pattern when boundary conditions and geometric models are symmetric on both sides. However, factors such as the asymmetrical air supply and staff activities which may cause the asymmetrical flow patterns in the cabin were not considered. A total of 15 passengers were seated in the aircraft cabin. The airsupply inlet of the cabin was on the side wall near the ceiling, while the exhaust outlet was near the floor. The passengers' skin temperature was set to $30.3{ }^{\circ} \mathrm{C}$, and the cabin walls were among 21 to $23{ }^{\circ} \mathrm{C}$. The air-supply flow rate was set as $9.4 \mathrm{~L} / \mathrm{s}$ per person, according to the recommendation provided by ASHRAE Standard 1612013 [14]. 


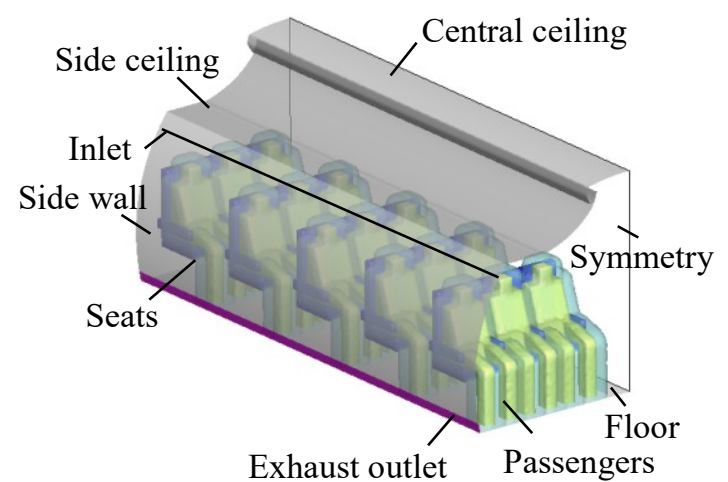

Fig. 2. Geometric model of a five-row aircraft cabin for inverse design of the air-supply parameters.

The air-supply parameters, including the air-supply opening size, air-supply temperature, and air-supply direction, were inversely determined. The design targets were the averaged air speed less than $0.2 \mathrm{~m} / \mathrm{s}$ in the head regions and less than $0.35 \mathrm{~m} / \mathrm{s}$ in the ankle regions; the area-averaged absolute value of the cabin predicted mean vote $\left(\left|\mathrm{PMV}_{\mathrm{c}}\right|_{\text {ave }}\right)$ in the surrounding surfaces at a distance of $0.1 \mathrm{~m}$ from the human body less than 0.3 ; and the area-averaged mean age of air in the head regions less than 120 seconds. Here, $\left|\mathrm{PMV}_{\mathrm{c}}\right|_{\text {ave }}$ means the average of the absolute $\mathrm{PMV}_{\mathrm{c}}$. The absolute operation was conducted first to calculate the deviation from the neutral $\mathrm{PMV}_{\mathrm{c}}$ for each passenger, followed by the average operation to evaluate the thermal comfort for passengers in five rows. The $\mathrm{PMV}_{\mathrm{c}}[15]$ was specially proposed for aircraft cabin with the reduced air pressure effect accounted for the eastern Asian passengers. In addition, the designs to obtain the minimum $\left|\mathrm{PMV}_{\mathrm{c}}\right|_{\text {ave }}$ and the minimum area-averaged mean age of air, respectively, were also provided in the study.

The initial parametric ranges when starting the design were set according to experience as: 1 to $3 \mathrm{~cm}$ for the air-supply opening size, $-45^{\circ}$ to $45^{\circ}$ for the air-supply direction, and $16{ }^{\circ} \mathrm{C}$ to $26{ }^{\circ} \mathrm{C}$ for the air-supply temperature. The air-supply direction was defined with respect to the horizontal, and it was positive if the direction was downward.

To solve for the thermo-flow fields under different air-supply parameters, the geometry as shown in Fig. 2, was created. Approximately 2.5 million combined tetrahedral and hexahedral grid cells were generated. The adopted grids were fine enough to reach the grid independence. The Fluent software was used to solve the thermos-flow. The RNG $k-\varepsilon$ model was utilized to resolve the flow turbulence. To well capture thermal buoyancy, the Boussinesq approximation was adopted to represent the varying density with temperature. In addition, the pressure staggering option (PRESTO) scheme was selected when discretizing the pressure term, while all the rest terms were discretized with the upwind scheme. The semi-implicit method for pressure linked equations (SIMPLE) scheme was used for pressure and velocity coupling. The convergence was judged to be satisfactory if the relative residual was small enough and there was no meaningful change in temperature, velocity, and mean age of air at a typical monitoring point with numerical iterations.

\section{Results}

\subsection{Typical thermo-flow distributions}

Fig. 3 shows the velocity, temperature, $\mathrm{PMV}_{\mathrm{c}}$ and mean age of air distributions for a case with $3.0 \mathrm{~cm}$ as the airsupply opening size, $20.0^{\circ}$ as the air-supply direction, and $26.0^{\circ} \mathrm{C}$ as the air-supply temperature. It can be seen that although the boundary conditions were identical for all rows, slight differences in the solved $\mathrm{PMV}_{\mathrm{c}}$ and mean age of air among different rows existed. The difference was attributed to flow turbulence and instability inside the aircraft cabin.

\subsection{Optimal air-supply parameters}

The CFD-based genetic algorithm started the initial design from eight random cases within the initial parametric ranges. These cases formed the first generation of the design toward the circumscribed parametric ranges. In the initial design stage, a total of 10 generations for crossing and mutation had been implemented, constituting 58 cases for full CFD simulation. The smaller rectangular frame in Fig. 4 shows the circumscribed parametric ranges by the genetic algorithm; while the larger frame surrounding the whole domain in the figure represents the initial parametric ranges provided by users. The genetic algorithm circumscribed the air-supply parameters into 1.0 to $3.0 \mathrm{~cm}, 5.0$ to $34.0^{\circ}$, and 23.0 to $26.0^{\circ} \mathrm{C}$. The removed parametric ranges were those beyond the design targets.

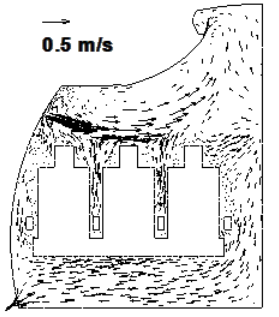

(a)

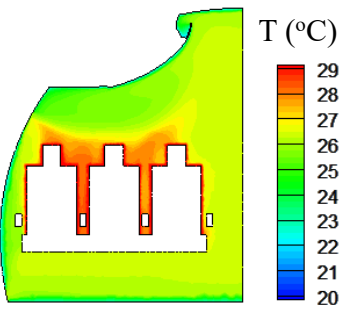

(b)

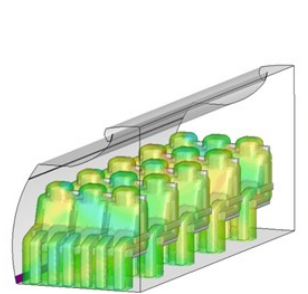

(c)

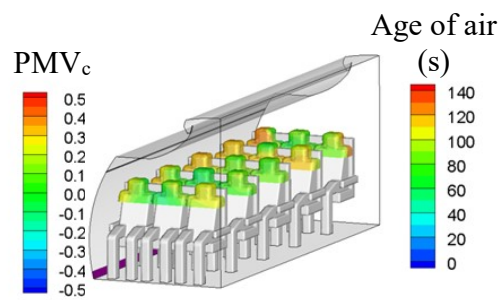

(d)
Fig. 3. Thermo-flow and environmental performance for a case with an opening size of $3.0 \mathrm{~cm}$, an air-supply direction of $20.0^{\circ}$, and an air-supply temperature of $26.0{ }^{\circ} \mathrm{C}$ : (a) air velocity vectors in one section across the passengers in the third row; (b) air temperatures in the same section; (c) $\mathrm{PMV}_{\mathrm{c}}$ on the 
surrounding regions at a distance of $0.1 \mathrm{~m}$ from the passengers; (d) mean age of air in the head zones.

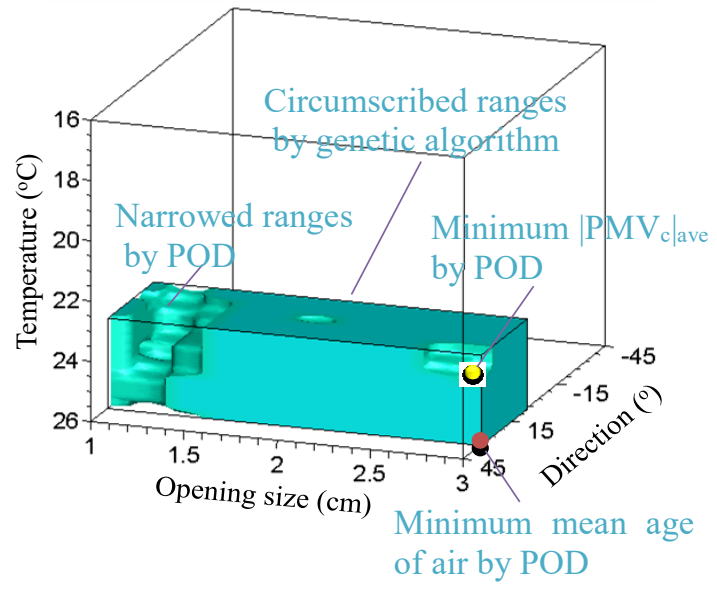

Fig. 4. Comparison of the initially defined parametric ranges (the larger frame surrounding the whole domain), the ranges circumscribed by the genetic algorithm (the smaller rectangular frame), the narrowed ranges determined by POD (the light blue, irregularly shaped regions), and the cases with minimum $\left|\mathrm{PMV}_{\mathrm{c}}\right|_{\text {ave }}$ (the upper yellow dot) and minimum mean age of air (the lower red dot) according to POD.

The circumscribed ranges by the genetic algorithm were provided to POD to extract the POD modes. Totally 45 cases were selected as representative cases, with discrete air-supply opening sizes of $1.0 \mathrm{~cm}, 1.5 \mathrm{~cm}$, $2.0 \mathrm{~cm}, 2.5 \mathrm{~cm}$, and $3.0 \mathrm{~cm}$, discrete air-supply directions of $5.0^{\circ}, 20.0^{\circ}$, and $34.0^{\circ}$, and discrete airsupply temperatures of $23.0{ }^{\circ} \mathrm{C}, 24.5{ }^{\circ} \mathrm{C}$, and $26.0{ }^{\circ} \mathrm{C}$. The light blue region in Fig. 4 is the optimized ranges that satisfied all of the four design targets according to the POD design. Some parameters were removed in this stage since they violated one or more design targets. Moreover, the air-supply parameters corresponding to the minimum $\left|\mathrm{PMV}_{\mathrm{c}}\right|_{\text {ave }}$ and the minimum area-averaged mean age of air were outputted, as shown by the yellow and red dots, respectively, in Fig. 4.

Table 2. Optimal designs obtained by the integrated method.

\begin{tabular}{|c|c|c|}
\hline Design criteria & $\begin{array}{c}\text { Case with } \\
\text { minimum } \\
\left|\mathbf{P M V}_{\mathrm{c}}\right|_{\text {ave }}\end{array}$ & $\begin{array}{c}\text { Case with } \\
\text { minimum } \\
\text { Age }_{\text {ave }}\end{array}$ \\
\hline Opening size & $2.7 \mathrm{~cm}$ & $3.0 \mathrm{~cm}$ \\
\hline Direction & $6.5^{\circ}$ & $32.2^{\circ}$ \\
\hline Temperature & $25.0^{\circ} \mathrm{C}$ & $26.0^{\circ} \mathrm{C}$ \\
\hline $\begin{array}{c}\text { Environmental } \\
\text { performances }\end{array}$ & $\mid \mathrm{PMV}_{\mathrm{c} \mid \mathrm{ave}}=0.09$ & $\mathrm{Age}_{\mathrm{ave}}=91.1 \mathrm{~s}$ \\
\hline
\end{tabular}

Finally, the above two optimal designs were inputted to the adjoint method for fine tuning. After five design cycles, i.e., five cases, the design with the minimum $\left|\mathrm{PMV}_{\mathrm{c}}\right|_{\text {ave }}$ was obtained in the third cycle. The corresponding $\left|\mathrm{PMV}_{\mathrm{c}}\right|_{\text {ave }}$ was 0.09 . The minimum mean age of air was $91.1 \mathrm{~s}$, also attained in the third design cycles. Table 2 summarizes the optimal air-supply parameters in terms of $\left|\mathrm{PMV}_{\mathrm{c}}\right|_{\text {ave }}$ and Age $\mathrm{Ave}_{\text {ave It shows }}$ that the air-supply parameters to reach the minimum $\left|\mathrm{PMV}_{\mathrm{c}}\right|_{\text {ave }}$ and $\mathrm{Age}_{\mathrm{ave}}$ are different. The users have to select their prefered design criterion to optimize a specific design.

\subsection{Justification of the method integration}

Table 3 lists the air-supply parameters to reach the minimum $\left|\mathrm{PMV}_{\mathrm{c}}\right|_{\text {ave }}$ in each stage. In the initial design, the minimum $\left|\mathrm{PMV}_{\mathrm{c}}\right|_{\text {ave }}$ for all of the 58 cases resolved by the genetic algorithm was 0.29. The minimum $\left|\mathrm{PMV}_{\mathrm{c}}\right|_{\text {ave }}$ was reduced into 0.09 by the POD. The minimum $\left|\mathrm{PMV}_{\mathrm{c}}\right|_{\text {ave }}$ obtained by the genetic algorithm was greater than that provided by the POD. This shows that the POD method provided a better design than the genetic algorithm. The ultimate fine design by the adjoint method produced the same $\left|\mathrm{PMV}_{\mathrm{c}}\right|_{\text {ave }}$ as the POD. However, the corresponding air-supply parameters differed slightly. The POD has provided a relatively good estimation of the optimal design for the adjoint method as the initial parameters.

Table 4 shows the minimum averaged mean age of air in the three stages. The Age ave was gradually reduced from $110.5 \mathrm{~s}$ in the initial design, to $93.0 \mathrm{~s}$ in the basic design, and finally to $91.1 \mathrm{~s}$ in the fine design. The integrated method improved the design stage by stage. The integration of the three methods was indeed necessary and valid.

Table 3. Cases with the minimum $\left|\mathrm{PMV}_{\mathrm{c}}\right|_{\text {ave }}$ obtained in each stage.

\begin{tabular}{|c|c|c|c|}
\hline Design stage & $\begin{array}{c}\text { Initial } \\
\text { design }\end{array}$ & $\begin{array}{c}\text { Basic } \\
\text { design }\end{array}$ & $\begin{array}{c}\text { Fine } \\
\text { design }\end{array}$ \\
\hline Opening size & $1.0 \mathrm{~cm}$ & $2.8 \mathrm{~cm}$ & $2.7 \mathrm{~cm}$ \\
\hline Direction & $21.8^{\circ}$ & $10.0^{\circ}$ & $6.5^{\circ}$ \\
\hline Temperature & $23.0^{\circ} \mathrm{C}$ & $24.8^{\circ} \mathrm{C}$ & $25.0^{\circ} \mathrm{C}$ \\
\hline$|\mathrm{PMV}|_{\mathrm{c}}$ ave & 0.29 & 0.09 & 0.09 \\
\hline
\end{tabular}

Table 4. Cases with the minimum averaged mean age of air obtained in each stage.

\begin{tabular}{|c|c|c|c|}
\hline Design stage & $\begin{array}{c}\text { Initial } \\
\text { design }\end{array}$ & $\begin{array}{c}\text { Basic } \\
\text { design }\end{array}$ & $\begin{array}{c}\text { Fine } \\
\text { design }\end{array}$ \\
\hline Opening size & $1.0 \mathrm{~cm}$ & $3.0 \mathrm{~cm}$ & $3.0 \mathrm{~cm}$ \\
\hline Direction & $21.8^{\circ}$ & $34.0^{\circ}$ & $32.2^{\circ}$ \\
\hline Temperature & $23.0^{\circ} \mathrm{C}$ & $26.0^{\circ} \mathrm{C}$ & $26.0^{\circ} \mathrm{C}$ \\
\hline Age $_{a v e}$ & $110.5 \mathrm{~s}$ & $93.0 \mathrm{~s}$ & $91.1 \mathrm{~s}$ \\
\hline
\end{tabular}

Regarding to the computing time, 58 full CFD cases were executed by the genetic algorithm, 45 cases by POD, and five cases by the adjoint method to optimize the design with the minimum $\left|\mathrm{PMV}_{\mathrm{c}}\right|_{\text {ave. }}$ Additional five cases were required to optimize the design with the minimum averaged mean age of air. Because the adjoint method solved both the Navier-Stokes equations and the adjoint equations in each design cycle, the computing load of an optimization case in the adjoint method was double of that for a full CFD-alone case. So a total of 123 equivalent CFD cases were required for the whole design to obtain the minimum $\left|\mathrm{PMV}_{\mathrm{c}}\right|_{\text {ave }}$ and the minimum mean age of air. However, if a user applied the 
genetic algorithm alone to find the comparative design, at least 200 full CFD cases were required. Therefore, the integrated method reduced the computing load by approximately $50 \%$ as compared with the CFD-based genetic algorithm method alone. Moreover, such a globally optimal design is extremely hard to be accomplished by the POD- or adjoint-based method alone.

\section{Discussion}

In the integrated design, the genetic algorithm solved the largest number of full CFD cases, and thus has a large potential to improve. Because the POD required only parametric ranges as the input, the genetic algortihm did not have to solve redundant cases. For example, some parametric sets that clearly produced poor designs could be eliminated early in the process. The bottleneck in accelerating the POD-based design was also the number of full CFD simulations to extract the POD modes. The POD used uniformly distributed parameters as characteristic cases, which might not be ideal. Intelligent schemes are required to identify representative characteristic cases, while the missing cases are reconstructed by interpolation. The integrated design was carried out sequentially stage by stage. The POD could omit some cases for full CFD simulation, if those cases were presented in the genetic algorithm. Likewise, the adjoint method could also skip cases resolved by the genetic algorithm and POD. In addition, the adjoint method utilized the steepest descent gradient for optimization. A superior strategy that might further speed up the search of the optimal design awaits to develop.

In this study, an air-supply temperature of $25{ }^{\circ} \mathrm{C}$ was obtained by the integrated design for the optimal $\left|P M V_{c}\right|_{\text {ave. }}$ Because heat sources such as electronic equipments and solar radiation were not considered in our investigation, the obtained air-supply temperatures may be higher than that in realistic flights. The cabin surface temperatures were fixed in the range from 21 to $23{ }^{\circ} \mathrm{C}$, which also made significant heat loss out of the cabin and thus required warm air supply to keep comfort. Further research may consider heat sources inside the cabin and set more practical thermal boundary conditions for cabin surfaces

\section{Conclusions}

This study proposed a strategy to integrate three different methods in cascades for inverse design of the air-supply parameters in an aircraft cabin. The air-supply opening sizes, directions, and temperatures to obtain the minimum $\left|\mathrm{PMV}_{\mathrm{c}}\right|_{\text {ave }}$ and the minium mean age of air were resolved. The results showed that the genetic algorithm was able to circumscribe parametric ranges for further design by the POD. The POD could estimate a good optimal design for each design criterion to the adjoint method. The integrated method improved the design stage by stage. The method integration was thus necessary and valid. Moreover, if the genetic algorithm alone was utilized to provide a comparative design as the integrated method, it would require at least double computing expense of the integrated strategy.

The work was supported by the National Key Basic Research and Development Program of China (the 973 Program, Grant: 2012CB720100) and the National Natural Science Foundation of China (Grant: 51622804).

\section{References}

1. J.H. Holland, Adaptation in natural and artificial systems: An introductory analysis with applications to biology, control, and artificial intelligence (the University of Michigan, Ann Arbor, 1975)

2. J.J. Grefenstette, IEEE T. Syst. Man Cyb. 16(1), 122-128 (1986)

3. M. Srinivas, L.M. Patnaik, IEEE T. Syst. Man Cyb. 24(4), 656-667 (1994)

4. Y. Xue., Z. Zhai, Q. Chen, Build. Environ. 64, 7784 (2013)

5. Z. Zhai, Y. Xue, Q. Chen, Build. Simul. 7(6) 661669 (2014)

6. J.L. Lumley, Atmospheric Turbulence and Radio Wave Propagation, Nauka Press, Moscow, 166-178 (1967)

7. Y. Wei, T. Zhang, S. Wang, Build. Environ. 96, 131-141 (2016)

8. Q. Chen, Z. Zhai, X. You, T. Zhang, Inverse design methods for the built environment (Taylor \& Francis, Abingdon, 2017)

9. C. Othmer, Int. J. Numer. Meth. Fl. 58(8), 861-877 (2008)

10. A. Jameson, J. Sci. Comput. 3(3), 233-260 (1988)

11. R.M. Neupauer, J.L. Wilson, Water Resour. Res. 35(11), 3389-3398 (1999)

12. W. Liu, R. Duan, C. Chen, C.H. Lin, Q. Chen, Energy Build. 104, 147-155 (2015)

13. K. Li, W. Xue, C. Xu, H. Su, Energy Build. 67, 3443 (2013)

14. ASHRAE Standard 161-2013. Air quality within commercial aircraft (ASHRAE Inc., Atlanta, GA, 2013)

15. W. Cui, Influencing factors and evaluation method of thermal comfort in commercial aircraft cabin $(\mathrm{Ph}$. D. Thesis, Tsinghua University, 2016) 